\title{
Link 11 Network Simulation Based on OMNeT++
}

\author{
Biao Fang, Gaoming Huang, Yongbin Wang, Jun Gao, Chengxu Feng, Zhen Li \\ Naval University of Engineering, 430033 \\ Wuhan, Hubei, China \\ Email: allan_fb@163.com
}

\begin{abstract}
In the life cycle of Tactical Data Link (TDL) system, the simulation is an indispensable key. This paper places emphasis upon a new object-oriented modular simulation framework-OMNeT++, and polling protocol of MAC layer is explored under its environment. Besides, TDL simulation model and Link11 network model based on the polling protocol are provided. The overall system performance such as network cycle, MAC port-to-port delay, packet delivery ratio and their analysis with network load were obtained. As is shown in simulation results, the network performance is fine, which could keep real time and reliability. It would be of a good value to the application and further research on TDL.
\end{abstract}

Keywords-TDL; Network Simulation; OMNeT++; Polling Protocol

\section{INTRODUCTION}

Modeling and simulation, which have been providing more and more important support in the whole life cycle of TDL, are becoming an indispensable key technique during the research and design of TDL. As early as the 60s last century, the U.S. Air Force had developed SAGE[1] system in order to simulate the TDL communication between the fighter plane and missile system for aerial defence.

Several researchers have studied the TDL network simulation models and performance evaluation under scheduled conditions below commercial network simulation tools, such as OPNET, QualNet. However these commercial tools are of high cost, the source codes are not available in educational version, which brings a lot of handicaps in the secondary programing. In this paper, a new discrete event simulation system-OMNeT $++[2,3,4,5]$ is proposed The purpose of the research was to analyze the effects of this open-source and modular simulation framework used on TDL simulation. Polling program andLink11 simulation model based on it are established, afterward, numerical experiments are designed and the performance is evaluated. The results we obtained demonstrate that this simulation framework is convenient and efficient for protocol modeling and large-scale topology building. Via Link11 network simulation, we can conclude that $\mathrm{OMNeT}++$ can be better used to realize complicated communication simulation and meet the need of digitalization war field provided with TDL, weapon platforms and wireless sensors.

This paper is organized as follows. Firstly, A brief background of the TDL system model is presented, the model of node, statistical, and polling protocol, including the detailed design of protocol are extended in Section II. Secondly, A simulation scenario under the environment is provided, including the whole set of Link11 network data processing based on $\mathrm{OMNeT}++$, then the designs are supported by an end-to-end simulation of a proof of the framework in Section III. Finally a conclusion is given in Section IV.

\section{TDL SYSTEM MODEL}

As to the simulation of TDL network, above all, we should simulate the access and assignment of channel resource, besides, to realize the simulation of message disposal and delivery. Therefore, the networking technique used to assign channel resource and the network architecture used to define protocols and interfaces which guarantee communication and cooperation among modules are three kinds of key technology in the TDL network simulation. A typical TDL system and function of components are shown in Fig.1.

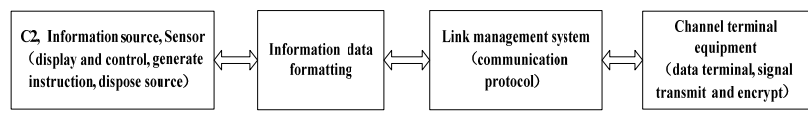

Fig.1. TDL system structure

On the basis of TDL structure and the layered reference model[6], we establish an abstract model of TDL, and corresponding layers are in parentheses (see Fig.2).

\begin{tabular}{|c|c|}
$\begin{array}{c}\text { Simulant } \mathrm{C} 2 \\
\text { (application and } \\
\text { service layer) }\end{array}$ & $\begin{array}{c}\text { Simulant networking } \\
\text { (network and link } \\
\text { layer) }\end{array}$ \\
\hline
\end{tabular}

Fig.2. Abstract TDL system structure

Simulant C2-answering for the generation of traffic, display and control, format disposal. Simulant networkinganswering for the simulation of communication protocols. Simulant channel-answering for the simulation of channel characteristics.

As shown in Fig.2 we can combine the three parts in one application program, making the coupling of modules tight. That is the idea of concentrated simulation model of TDL network, which can be realized by network simulation tools like OPNET, QualNet. This paper introduces a new objectoriented, open-source, modular simulation tool-OMNeT++, the predominance of open source and program style similar with $\mathrm{C}++$ are of great assistance in the design and modification of network architecture and protocols.

OMNeT++ itself is not a simulator of anything concrete, but it rather provides infrastructure and tools for writing simulations. One of fundamental ingredients of this infrastructure is a compositive architecture for simulation models. Concrete simulation can be sustained by module architectures such as Mobility Framework(MF)[7,8] and 
INET Framework. These frameworks are independent with $\mathrm{OMNeT}++$ and have their own release periods. The latest edition of MF is $2.0 \mathrm{p} 3$, which is used mostly in our study.

\section{A. Node Model}

The node model of TDL is an independent compound module, which covers all layers of TDL network architecture. Each module in the node model fulfills the function of each layer of the architecture. Every protocol layer corresponds to a module. We founded the model below MF[9] by extending functions and modifying codes in source files. The model is shown in Fig.3.

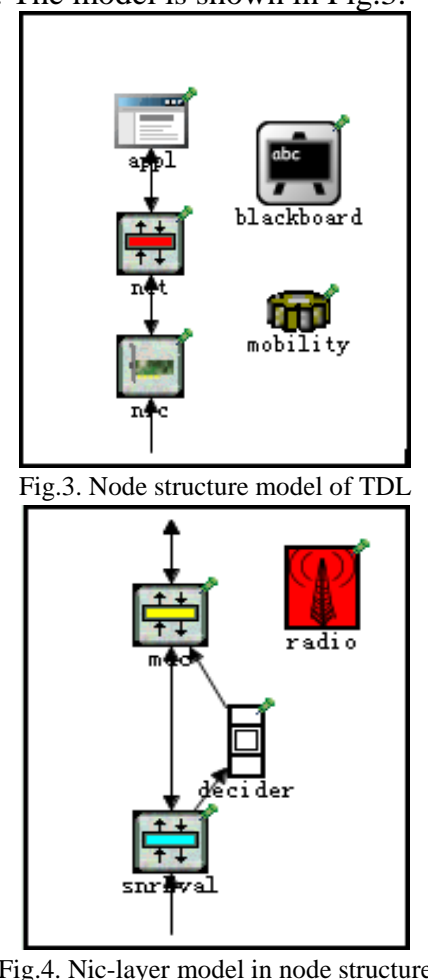

The node model is composed of an application layer module(appl), a network layer module(net), a network interface layer module(nic), a mobility module (mobility) and a manage module(blackboard). In particular, the nic module is composed of a medium access layer module(mac), a signal-to-noise evaluation module(snrEval), a decider module(decider) and a wireless diffusion module(radio). Fig. 4 shows the structure of the compound module nic.

The appl is used to generate traffic such as broadcast message or polling message. In the Link11 simulation network, tasks of appl are to open or close files when simulation running starts or closes, to calculate the network entrance time, to calculate the network cycle of a polling round. The task of net is to maintain the network route, while in a single link network there is no need to route, so the net takes charge of member list maintenance. Tasks of mac are to control channel accessing, to calculate the packet delivery ratio and port-to-port delay, and polling codes concentrate in this module. The snrEval is used to calculate the signal-to-noise to determine whether to send the packet up to decider. The radio is a wireless diffusion model possessing the characteristic of threshold cut-off. The process to receive a packet divides two steps: (1) If the signal power level is under the signal attenuation threshold, the signal will be treated as noise to discard, otherwise send the packet from snrEval to decider. (2) If the signal power level is under the receiver sensitivity, the signal will be treated as a false packet and send the packet to mac, otherwise send the packet to mac as right. The mobility is to control the movement of nodes. There have already been seven movement models in MF (LinearMobility, ConstSpeedMobility, CircleMobility, RecTanguleMobility, TurtleMobility, MassMobility, BonnMotionMobility)[7]. The function of blackboard is to exchange data for crossing layers. It is realized by the release-subscribe mechanism.

\section{B. Polling Protocol Model}

In $[7,10]$, the means of protocol extension had been studied, the polling protocol model is established below MF.

The polling program is mainly concentrated in the control station. The program consists of three self-message timers(a startup timer for network entrance SEND_ BROADCAST_TIMER, a closure timer for network exit BROADCAST_REPLY_TIMEOUT, a timer for polling reply overtime POLL_REPLY_TIMEOUT), four messages (BROADCAST_MESSAGE, POLL_MESSAGE, POLL_ REPLY, BROADCAST_REPLY_MESSAGE ) and five functions (sendBroadcast() is to send broadcast message, sendPoll() is to send polling message, pollNext() is to poll next member, initialize() and finish() is to indicate the simulation begin and end).

The process of Link 11 simulation network based on polling protocol is as following:

1) Initialization, publish and subscribe the parameters, startup the network entrance timer SEND_ BROADCAST_TIMER.

2) Call handleSelfMsg() to deal with the SEND_ BROADCAST_TIMER when it times up. Then generate BROADCAST_MESSAGE and pack it to send, startup BROADCĀST_REPLY_TIMEOUT to restrict broadcast time.

3) Receive BOADCAST_REPLY_MESSAGE from passive stations, add the station to the polling member list.

4) BROADCAST_REPLY_TIMEOUT times up, start to poll according to the member list, call sendPoll() to send POLL_MESSAGE, and startup the timer POLL_REPLY_TIMEOUT. If receiving POLL_ REPLY from the passive station, the control station cancels timer POLL_REPLY_TIMEOUT, otherwise removes the passive station from the polling list.

5) Receive POLL_REPLY, the control station calls pollNext() to check the member list. If the round is not over, it will poll the next member, otherwise start a new round.

6) Simulation times up or pauses, call finish() to track record and finish the simulation. 


\section{PERFORMANCE EVAluATION}

\section{A. Simulation Method and Environment}

The performance of different stations in network and the overall performance of network are compared by means of landscape orientation and vertical orientation. The number of passive stations is the dividing line. Firstly we experimented on a scene with 10 passive stations. The control station sent broadcast and polling packets, and the size of packets and the speed of stations were volatile. Tracked the record of MAC port-to-port delay, packet delivery ratio, network cycle as well as channel utilization during simulation. Then we made a series of simulations by increasing 5 passive stations each time until the network load reached 50. The network performance trend along with the change of network load would be obtained finally.

Grand simulation parameters for Link 11 networkSimulation Area: 2000m*2000m; Simulation Time: 1000s; Carrier Frequency: 24MHz; Number of Passive stations: 10 (increase 5 each simulation until 50); Node Speed: Control Station-fixed, Passive Station-airplane (200m/s), vessel $(15 \mathrm{~m} / \mathrm{s})$, vehicle $(10 \mathrm{~m} / \mathrm{s})$, soldier $(1 \mathrm{~m} / \mathrm{s})$; Mobility Pattern: LinearMobility; MAC protocol: Polling; MAC Queue Length: 10; Broadcast Reply Timeout: 45s; Polling Reply Timeout: 15s; Transmission bit rate: 1800 b/s; Transmission Power:100W; Receiver Sensitivity:-100dBmW; Minimum Signal Attenuation Threshold: -120dBmW.

The .ini file contains the full configuration of simulation network.

As an example of 10 passive stations, the simulation animation is shown in Fig.5.

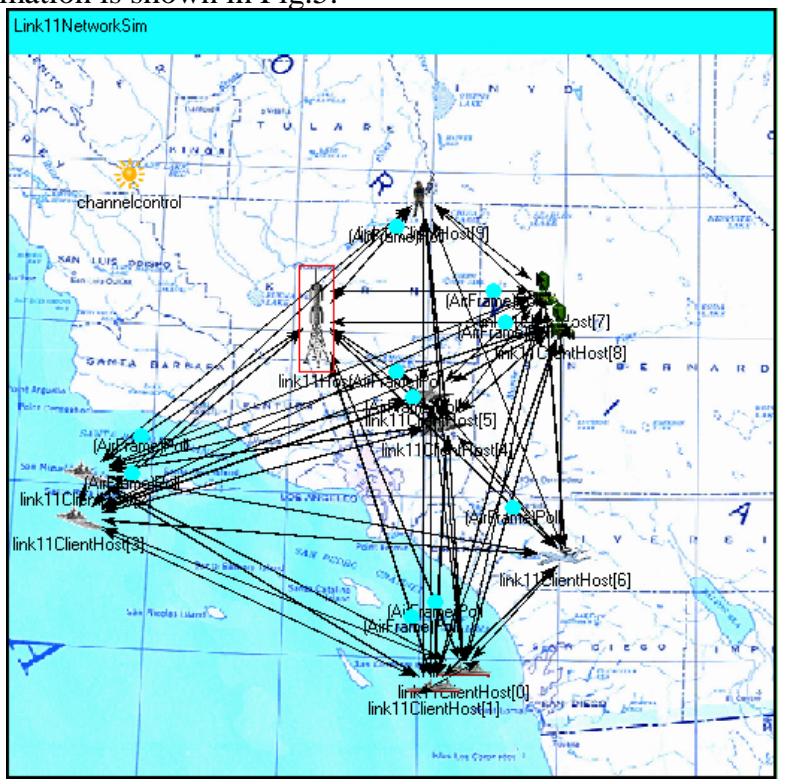

Fig.5. Simulation running animation

\section{B. Results and Analysis}

In this section we made use of the analyzer[2,11] OMNeT++ provided to investigate and analysis parameters of network performance obtained during simulations.
In a TDL network, port-to-port delay is a period of time from a packet sent to channel till the MAC layer of sink receives the packet. It represents the real-time capability of network. As military communication, TDL demands well behavior of real time to assure the efficiency of communication.

Fig.6 shows port-to-port delay of 10 passive stations from node 0 to 9 and the control station (blue denotes maximum, green denotes minimum, red denotes mean and yellow denotes standard deviation). As is shown, the average delay concentrates around 2s. Land nodes (node 7, 8 and 9) behave better than other nodes in every item value, which is mostly as a result of the considerably stabilization and low speed. Airplane nodes 4, 5, 6 behave worse. Increasing speed and changing path cause timeout and channel condition worsening which lead to longer port-to-port delay.

To study the effect of reliability, we measure the performance of packet delivery ratio of all nodes. As is shown in Fig.7, nodes 1, 4, 5, 6 are below other nodes in performance. That is because increasing speed leads to an increasing frequency of dropping packets. Although the speed of vessel node 1 is fixed at only $15 \mathrm{~m} / \mathrm{s}$, its mobility distance is away from the center which assists the decline in capacity. As a whole, the packet delivery ratios of all are over $60 \%$, which ensures the reliability of network basically.

The collision and error of packets will result on the waste of channel resource. The performance of MAC protocol can be reflected by the degree of waste. Channel utilization is defined by the ratio of the time used to deliver packets successfully and the time used in all. From the filed of information theory, it also can be defined by the ratio of network throughput which denotes the amount of information delivering all right and channel capacity which denotes the Shannon limit.

In Fig.8 we can see how the channel utilization increases with the number of passive stations. Channel utilization goes up almost linearly until 40 when it reaches an extremum $68.52 \%$, then the network starts showing signs of fluctuation around $65 \%$. This scenario suggests that the optimal network load is about 40 and the maximum of channel utilization is about $70 \%$, when the network throughput is maximal.

The network cycle of Link 11 is the period of time for the control station polling all members. The length is due to the number of members, the amount of information, the geographical distributing and so on. Fig.9 shows the rule of network cycle with the network load. When the number of passive stations is below 35, the network cycle goes up gradually in linearity and the gradient is $5.11 \mathrm{~s} /$ node. But the network cycle changes suddenly after 35 , the gradient increases to 8.67s/node dramatically. The slow increase is in respect that the network throughput is still under network capacity, the accession of passive stations does not have too much influence with the cycle. The rapid increase is in respect that the network throughput is near to the maximum, the increased traffic caused by accession of passive stations must need more transmission time. The transformation of curve suggests the optimal network load is between 35 and 40, which agrees with Fig.8. 


\section{CONCLUSIONS AND FUTURE WORK}

TDL system simulation comes down to kinds of hardware, software and practical equipments. The main question is how to combine these resources with simulation technique organically and efficiently. The paper researched the simulation implement below $\mathrm{OMNeT}++$ and Mobility Framework, founded the TDL system simulation model, compiled polling program, established Link 11 simulation model based on polling protocol, and evaluated their performance with built-in statistic modules. The results show that the simulation means are feasible and universal. Arguably, simulation results are not a total representation of actual TDL network performance, but it is a key to study and verify the MAC algorithm performances and its behavior. Such study is essential to aid the design of TDL network frame.

Our future work aims to consider on other MAC protocols, multi-Links network and distributed simulation with OMNeT++. On the other hand, to associate with MATLAB for Physical layer simulation can also be studied.

\section{REFERENCES}

[1] Mosera V M. JTIDS Integrated Siting Tool (JIST) technology overview [C]. Military Communication Conference 1992. New Jersey: IEEE, 1992: 883-887.

[2] OMNeT++ Home Page. http://www.omnetpp.org [accessed on December 2011].

[3] Varga A. OMNeT++ Discrete Event Simulation System Version 4.1 User Manual [EB/ OL]. http://www.omnetpp.org. [accessed on March, 2011].

[4] Varga A. OMNeT++ Development User Guide [EB/OL]. http://www. omnetpp.org. [accessed on March, 2011]

[5] Varga A, Hornig R. An Overview of the OMNeT++ Simulation Environment. In Proceedings of the First International Conference on Simulation Tools and Techniques for Communications, Networks and Systems (SIMUTools2008'), March, 2008.

[6] Wilson W. Applying Layering Principles to Legacy Systems: Link16 as a Case Study[EB/OL]. http://ieeexplore.ieee.org.

[7] Lobbers M, Willkomm D. A Mobility Framework for OMNeT++ User Manual Version 2.0[EB/OL]. http://www.omnetpp.org. [accessed on March, 2011].

[8] Camp T, Boleng J, Davies V. A Survey of Mobility Models for Ad Hoc Network Research[J]. Wireless Communications\&Mobile Computing, 2002, 2(5): 483-502.

[9] Mobility Framework[EB/OL]. http://mobility-fw.sourceforge.net [accessed on March, 2011].

[10] W. Drytkiewicz, S. Sroka, V. Handziski, A. Koepke, and H. Karl, A Mobility Framework for OMNeT++.3rd International OMNeT++ Workshop, 2003.

[11] Sunfast,TicToc Tutoria for OMNeT++[DB/OL]. http://sunfast.cublog. cn. [accessed on March, 2011]

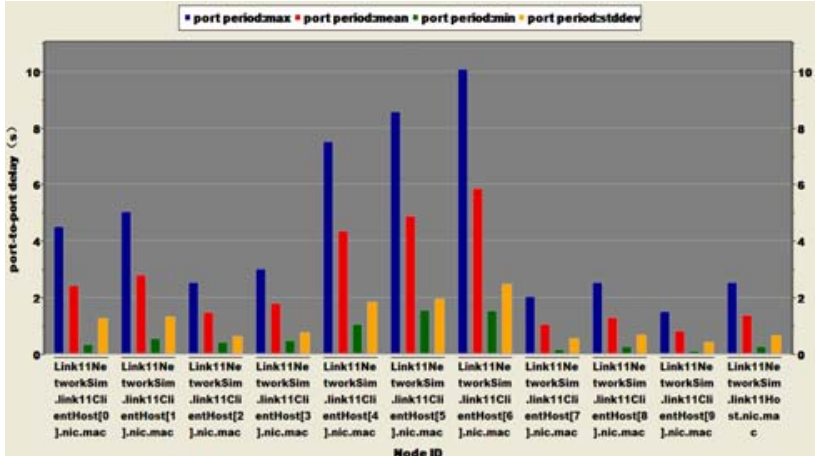

Fig.6 Port-to-port delay of MAC layer
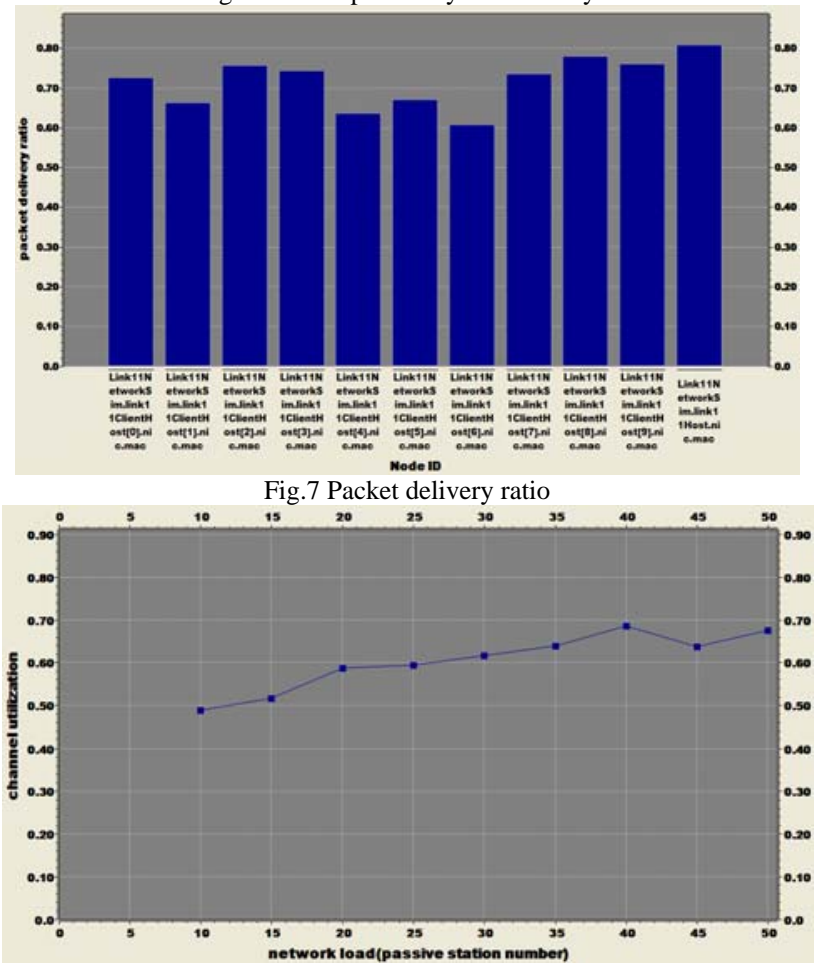

Fig.8 Channel utilization vs. Network load

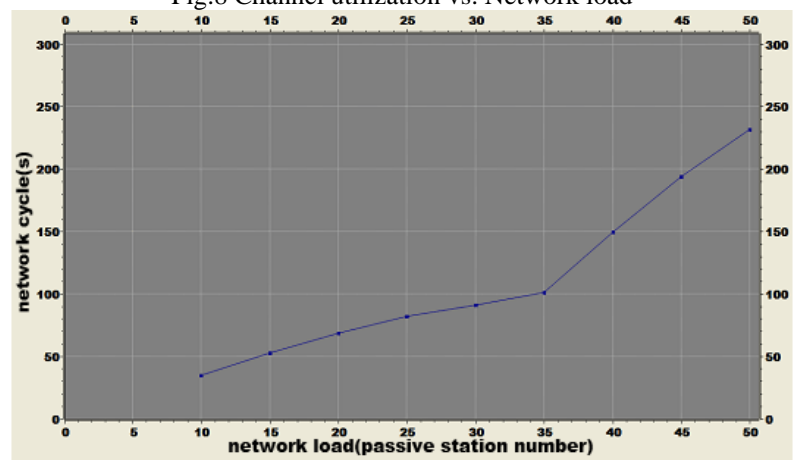

Fig.9 Network cycle vs. Network load 